\title{
Environmental Surveillance for Poliovirus in Polio High Risk States of Nigeria, 2011- 2012
}

\author{
Goitom Weldegebriel ${ }^{1,}$, , Adekunle Adeneji ${ }^{2}$, Alex Gasasira ${ }^{1}$, David Okello ${ }^{1}$, Chris Elemuwa ${ }^{3}$, \\ Asghar Humayun ${ }^{4}$, Ousmane Diop ${ }^{5}$, Rakoto Mala ${ }^{6}$ \\ ${ }^{1}$ World Health Organization, Nigeria, Abuja \\ ${ }^{2}$ University of Ibadan, department of Virology, Nigeria \\ ${ }^{3}$ National Primary Health Care Development Agency, Nigeria, Abuja \\ ${ }^{4}$ World Health Organization, Regional Office for the Eastern Mediterranean, Egypt, Cairo \\ ${ }^{5}$ World Health Organization, Geneva \\ ${ }^{6}$ World Health Organization, Regional Office for Africa, Brazzaville
}

\section{Email address:}

weldegebrielg@who.int (G. Weldegebriel)

\section{To cite this article:}

Goitom Weldegebriel, Adekunle Adeneji, Alex Gasasira, David Okello, Chris Elemuwa, Ashgar Humayun, Ousmane Diop, Rakoto Mala. Environmental Surveillance for Poliovirus in Polio High Risk States of Nigeria, 2011- 2012. Science Journal of Public Health. Vol. 3, No. 5, 2015, pp. 655-663. doi: 10.11648/j.sjph.20150305.20

\begin{abstract}
Introduction: Nigeria is one of the three polio endemic countries in the world along Pakistan and Afghanistan. The detection of persons with Acute Flaccid Paralysis (AFP) and testing of stool specimens from these patients is the surveillance standard for detection of poliovirus. World Health Organization recommends complementary surveillance by testing sewage samples and stool of healthy children. Kano is the epi-center of polio in Nigeria. Environmental surveillance was introduced in June 2011 in Kano State and in April 2012 in Sokoto State. Methods: Grab method was used to collect sewage samples by trained environmental health workers. The samples were tested in Ibadan Polio Laboratory which is part of the Global Polio Laboratory Network. The Samples were concentrated using the two-phase separation method. Isolation of Poliovirus was carried out in RD and L20B cell lines. Poliovirus identification was done using the micro neutralization techniques. Results: In Kano State, from week 28 of 2011 to week 52 of 2012, a total of 60 samples were collected. In Sokoto State, from week $13-$ 52 of 2012, a total of 80 sewage samples were collected from four sewage sites. In Kano and Sokoto, 62 and 93 single or mixed isolates were detected from the samples. In Kano, $39(63 \%)$ of the isolates were Sabin viruses, $16(26 \%)$ were circulating vaccine derived polio viruses type 2 (cVDPV2), 2 (3\%) were wild polio virus type 1 (WPV1), 4 (6\%) were non polio enteroviruses (NPENT) and 1 (3\%) were wild polio virus type 3 (WPV3). In Sokoto, 33 (35\%) of the isolates were cVDPV2, 27 (29\%) were Sabin viruses, 16 (17\%) were wild virus type 1 and $17(18 \%)$ were non polio enteroviruses. No wild virus type 3 was detected from AFP cases and environmental samples in Sokoto State in 2012. Conclusion: The results confirm the prevailing immunity gap in polio high risk areas of Nigeria and pronounced immunity gap against type 2 polio virus in Sokoto. Long distance travelers such as nomads play important role in disseminating poliovirus. Special focus should be given to reach and vaccinate such underserved and migrant communities. In addition to the national campaigns with bivalent oral polio vaccine (bOPV) and trivalent oral polio vaccine (tOPV), an aggressive strategy should be adopted to mop up any detection of cVDPV in cases, contacts, or the environment.
\end{abstract}

Key words: Polio, Environmental Surveillance, Kano, Sokoto

\section{Introduction}

Enteroviruses are a subgroup of the picornaviridae family. They are classified into poliovirus (3 sero types) and new enteroviruses [1, 2]. Transmission of these viruses is usually by fecal-oral [3].
The risk of infection is directly correlated with poor hygiene and poor sanitation and overcrowding, typically among inadequately vaccinated populations [4].

The detection of persons with Acute Flaccid Paralysis 
(AFP) and laboratory testing of stool specimens from these patients is the surveillance standard for global polio eradication. Reports of findings on lineages of indigenous wild virus in an endemic country nearly 3 years after last virus isolation by AFP surveillance has stimulated interest in use of environmental surveillance in developing countries [5].

Just one in 1,000 children infected by type 3 polio becomes paralyzed by the virus, meaning 999 out of 1,000 children carry the virus silently, infecting entire communities. Similarly, only one in 200 children infected with type 1 polio becomes paralyzed. Sewage sampling allows the polio eradication initiative to clearly determine whether the virus is circulating in what otherwise appears to be a healthy town or village [6].

After eradication of Wild Polio Virus (WPV) from AFP cases in high risk areas, World Health Organization (WHO) recommends complementary surveillance by using sewage sample and stool of healthy children [7].

Supplementary environmental surveillance has been valuable in India, where low-level transmission has occurred as well as in Pakistan, where substantial AFP surveillance indicators have met targets but virologic analysis indicated major gaps in detection [8].Currently, plans are being made to implement environmental sampling in Nigeria in 2011. As fewer circulating WPVs are detected, the role of environmental sampling will increase; in addition to its use for detection of potential vaccine derived polio viruses (VDPVs). Monitoring of surveillance performance indicators at Sub national levels continues to be critical to identify surveillance gaps that might allow WPV circulation to be missed in certain areas or subpopulations. To achieve polio eradication, efforts are needed to further strengthen AFP surveillance, implement targeted environmental surveillance, and ensure that the Global Polio Laboratory Network (GPLN) quality is maintained [8].

The validity and important roles of environmental surveillance has also been reported in Iran [9] and in Israel [10]. Environmental sampling has become a key strategy in the hunt for the final reservoirs of polio globally.

Nigeria has been a major reservoir for transmission of polio elsewhere. Since 2003, WPV of Nigeria origin has been imported into 25 countries, and many countries in the West and Central Africa have had repeated importations [11]. In 2004, polio cases caused by WPV originating from Northern Nigeria were reported in 11 countries - Benin, Botswana, Cameroun, Guinea, Mali, Saudi Arabia, Burkina Faso, Central African Republic, Chad, Cote d'Ivoire, and Sudan-reestablishing transmission in the latter five countries [12].

From the 125 countries infected by polio when the Global Polio Eradication Initiative (GPEI) began, in 2012, there were three left that have never stopped transmission. India was removed from the polio endemic countries in $\mathrm{Feb}$ 2012 [13]. Nigeria is one of the remaining three polio endemic countries in the world together with Afghanistan and Pakistan.

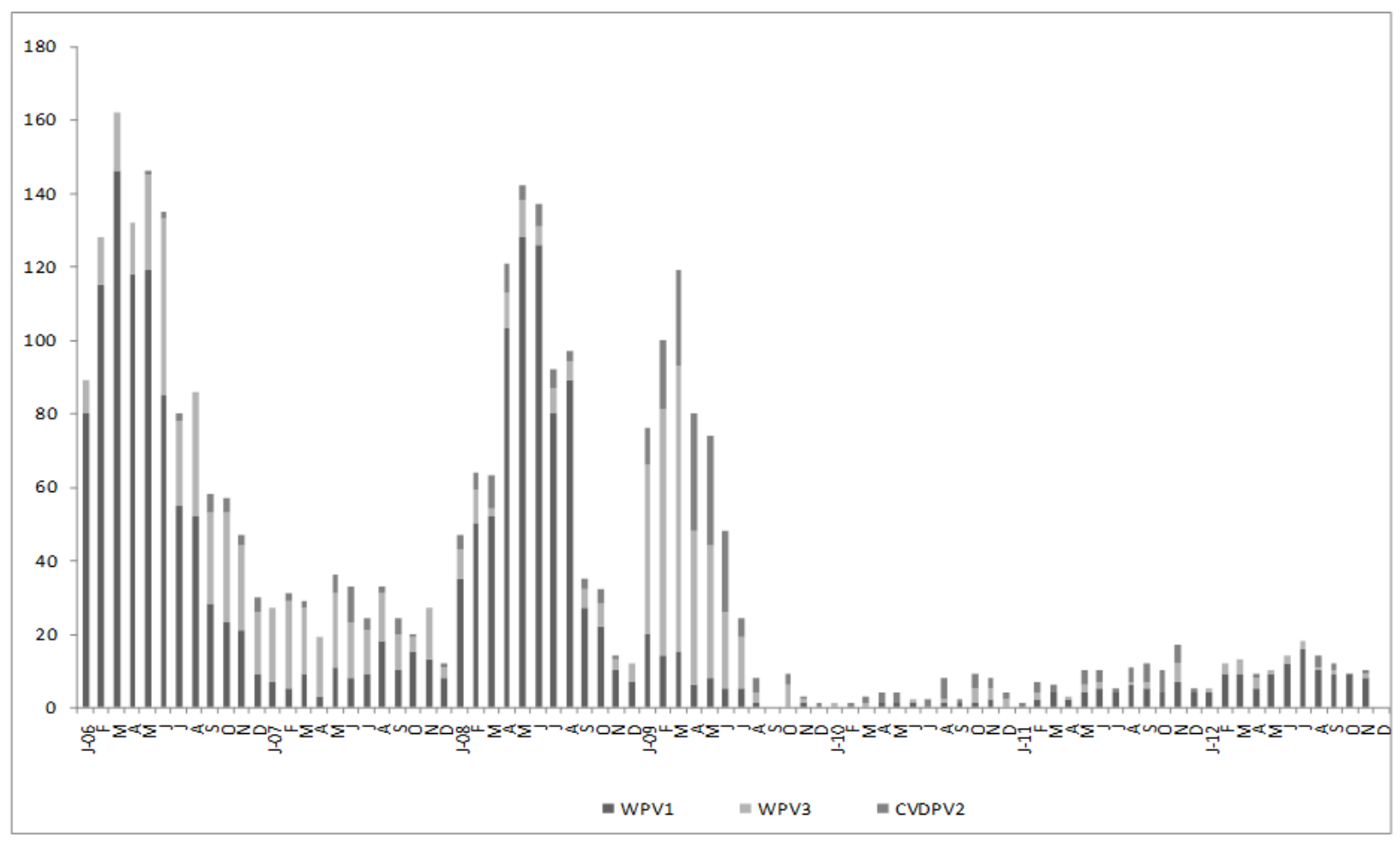

Figure 1. Poliovirus by month, 2006 - Dec 2012, Nigeria. Source: National AFP database, FMoH, Nigeria [14] 


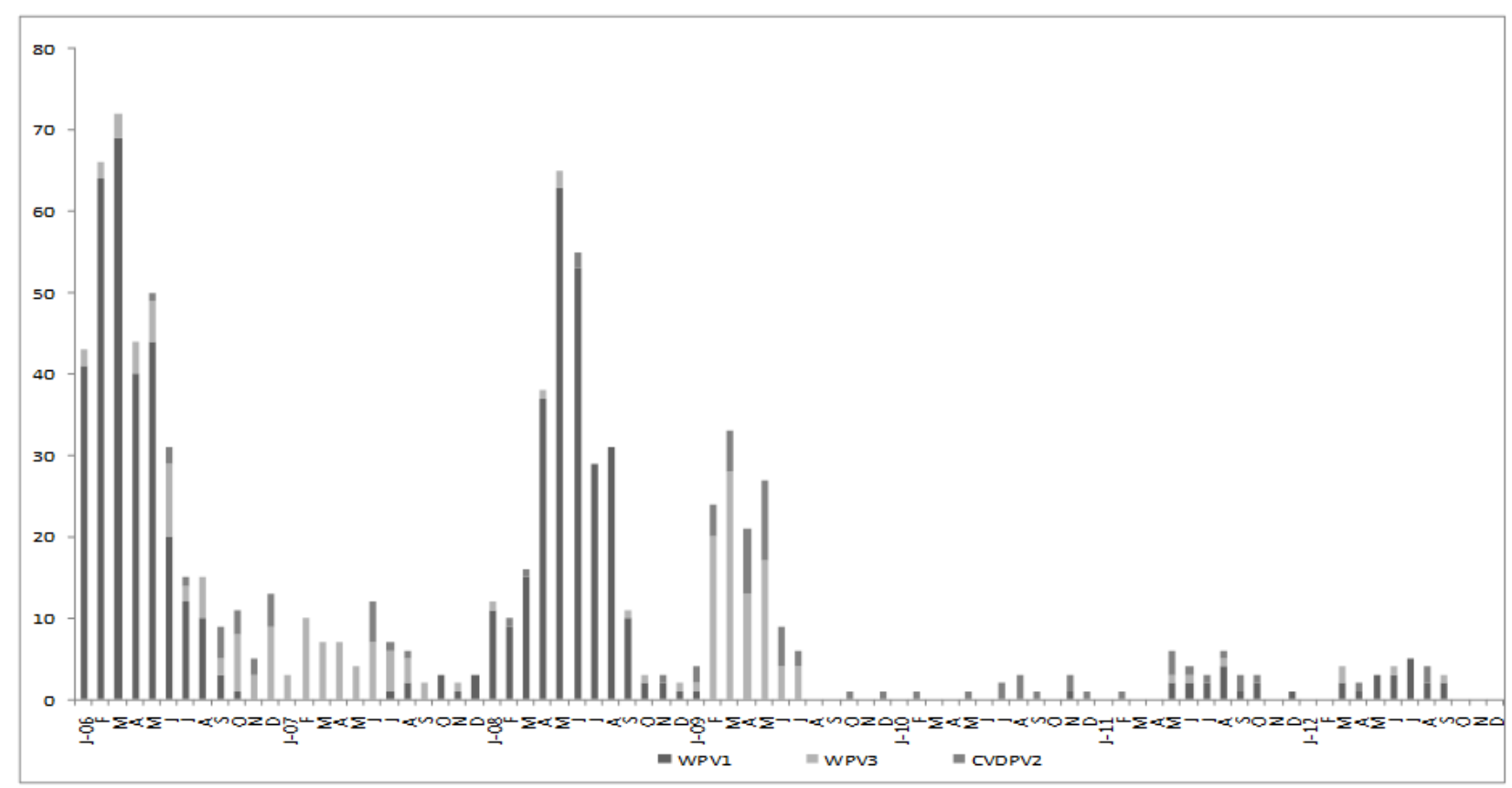

Figure 2. Poliovirus by month, 2006 - Sep 2012, Kano State, Nigeria. Source: National AFP database, Federal Ministry of health, FMoH, Nigeria [14]

From 2006 to 2011, Nigeria reported 583, 285, 798, 388, 21 and 62 Wild polioviruses type 1 and 3 [14]. A grouping of concurrent cVDPV2 outbreaks began in Nigeria in 2005 [10]. Since 2005, VDPV type 2 is being detected in the Northern States of the country. From 2005-2011, the country reported $3,22,71,66,154,27,34$ cVDPV2 cases. In 2012, Nigeria reported 8 cVDPV2 cases from 3 States, Kano (3], Sokoto (3) and Kebbi (2) [14]. Niger 2006, Niger 2009, Niger 2010, Chad 2010 cVDPVs are linked to the Nigeria outbreak [15].

Kano State is located in the Northern part of Nigeria and is known as the epicenter of polio in Nigeria. Between the year 2006 to 2011, Kano reported a total of 1,032 WPV cases (583 in 2006, 59 in 2007, 12 in 2008, 139 in 2009, 1 in 2010 and 17 in 2011). Following the uninterrupted transmission of poliovirus circulation in Kano State, no single WPV was reported in 12 months rolling period of August 2009 to August 2010; while 9 cVDPV2 cases were detected from AFP cases (figure 2).

\section{Genomic Sequence Analysis}

To investigate transmission of links, track international spread and estimate the duration of circulation, the nucleotide sequence of the VP1 region of each WPV and vaccine-derived poliovirus isolate is analyzed [8]. The genetic diversity of WPV1 (reflected by the number of chains of transmission and genetic clusters) decreased substantially during 2010 - 2011 in Nigeria. In 2010, 4 WPV1 genetic clusters were observed compared to 19 in 2009. However, $13 / 19$ (68\%) WPV1 isolates tested were $>1.5 \%$ divergent (that is, there was much less genetic linkage than expected with sensitive AFP surveillance), and 2/5 (40\%) WPV1 clusters observed in 2011 were not detected by AFP surveillance in 2010. In 2010, 4 WPV3 clusters were observed compared with 20 in 2009. However, 8/18 (44\%)
WPV3 isolates tested during 2010-2011 were $>1.5 \%$ divergent. Among $36 \mathrm{cVDPV} 2$ isolates tested during 20102011, 15/23 (64\%) were $>1.5 \%$ divergent [11].

In Nigeria, AFP surveillance targets have continued to be met in all States. However, virological data indicate that surveillance is not sufficiently sensitive to detect all chains of WPV transmission in a timely manner $[11,16]$.

The Expert Review Committee (ERC) on polio eradication and routine immunization in Nigeria, in its 19th meeting, 2224 March 2010, recommended the comprehensive assessment of the potential for supplemental environmental surveillance in key areas such as Kano Municipal [15]. In March 2012, the ERC in its 23rd meetings recommended the scale up of environmental surveillance in to another high risk State [16]. Accordingly, the environmental surveillance was scaled up in to Sokoto State. Sokoto is located in the Northern part of the country and is one of the polio high risk States. From 2006 - 2011, Sokoto State reported a total of 122 cases (41 in 2006, 26 in 2007, 28 in 2008, 16 in 2009, 4 in 2010 and 7 in 2011). In 2012, Sokoto reported 8 WPV1. Sokoto reported the first outbreak of cVDPV in 2007 with 30 cases. Between 2007 and 2011, 47 cVDPV2 cases were reported ( 30 in 2007, 8 in 2008, 5 in 2009, 3 in 2010, one case in 2011). In 2012, three cVDPV2 were detected from AFP sample in Sokoto State. The monthly situation of WPV and cVDPV2 in Sokoto State between 2006 - December 2012 is shown in Figure 3.

In response to the ERC recommendation, WHO Nigeria working with the National Primary Health Care Development Agency (NPHCDA), constituted a team of experts to visit Kano State. The team of experts comprised Public Health Experts and virologists from WHO and as well as local environmental experts from the Kano Ministry of Environment. In April 2012, the environmental surveillance 
was expanded into another polio high risk, Sokoto State, building on the experience and applying similar approaches to that of Kano.
This report summarizes the findings of the environmental surveillance in Kano and Sokoto States.

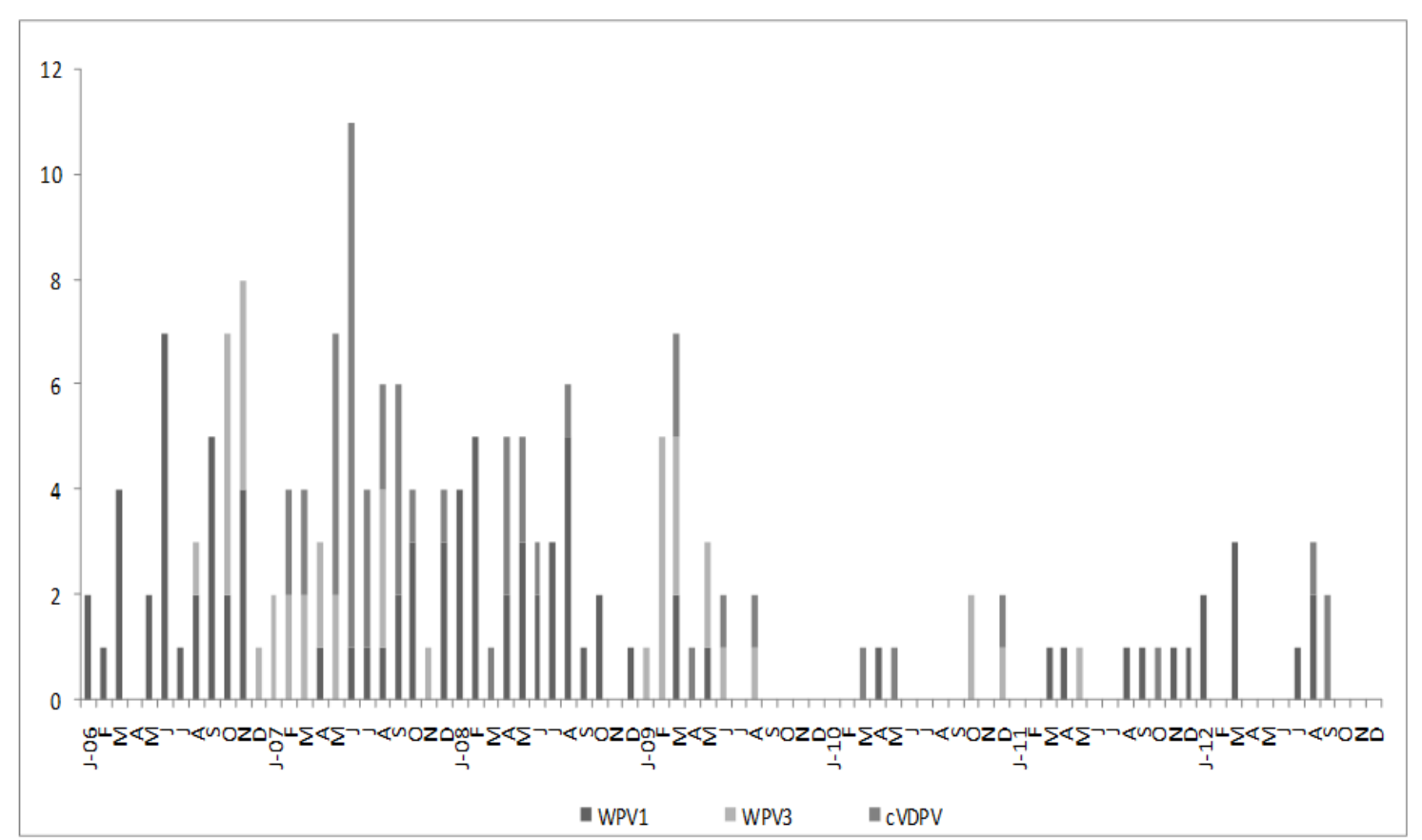

Figure 3. Poliovirus by month, 2006 - Sep 2012, Sokoto State Source: National AFP database, Federal Ministry of health (FMoH), Nigeria [14]

\section{Methods}

\subsection{The Selection of Sampling Sites}

Kano State was selected for the initial assessment because of the persistent transmission of wild polio viruses that the state experienced pre -2009 and as recommended by the $19^{\text {th }}$ ERC. In order to select the sampling sites in relation to the LGAs in Kano State, the following criteria were used:

- Whether or not the LGA was classified as a high risk LGAs based on existing data (population density and high risk population, sanitation, living conditions, routine immunization and SIAs coverage).

- Presence of a sewage disposal channels which receives waste from substantial proportion of the population in the catchment area, with a minimum amount of waste coming from other areas.

- Absence of industrial waste in the proposed site or canal.

- Access to this sewage to take a sample.

At the beginning of the assessment mission, the technical team met to discuss the possible LGAs that will be targeted in the setting up of the environmental surveillance based on the above criteria. Eight high risk LGAs in Kano State were identified as suitable LGAs for the setting up of environmental surveillance. Prior to this decision, information on the sanitation situation in the whole State had been obtained from the State Ministry of Environment by officers from the Ministry of Health.

There was no organized/functioning sewage wastewater disposal/treatment system in Kano State; however, there were remnants of 5 sewage pumping stations, which were no more functional. The population used different methods of sewage drainage namely: Soak away system, drainage of waste through pipe into main open sewage channel, direct disposal of waste into the open sewage channel and open disposal of fecal material. Despite the various methods of waste disposal identified, it was observed that around $60 \%$ of population waste was through the open sewage channel thus making it possible to collect good quality multiple samples for the purpose of environmental sampling.

There was one major sewage channel referred to as Jakara, which passes through the city and is the sole drainage system for the identified high risk Local Government Areas (LGAs). The drainage is mainly through open drains or through pipes into this nulla. It didn't have a smooth flow because all kind of waste material (shopping bags, dead animals, cloth, straws etc) were thrown into it, at places it is totally or partially blocked and wastewater was flowing below or overflowing from the side of blockade. It didn't have concrete walls and with varied width at different places, and it narrowed while passing through densely populated areas.

A member of technical team was tasked to observe the early morning flow of sewage in the main channel and noted that maximum flow of sewage water occurred between 06:00- 07:00 hours. For the purpose of planning, it was concluded that the best time for collection of samples would have to be between 06:00 and 07:00 hours.

The technical team inspected the main sewage channel in Kano State. This channel, the Jakara channel receives sewage via smaller and medium size sewage channels that drain 
residential areas of the various LGA in the State. Potential sampling sites were identified at the junctions between the Jakara channel and the smaller/medium size channels based on the above mentioned criteria.

Based on available data/field visits, risk factors, risk population epidemiology, the following three potential sites were identified for the initial setting up of the environmental surveillance in Kano State: 1. Jakara Police Station Site 2. Kurna Masallaci Site 3. Gogau Site.

In Sokoto State, similar methods described above were followed and used to identify four sites as sample collection sites in the Sokoto metropolis. The selected sites were 1. Kofar Dundaye, 2. Kofar Kware 3. Tudun Wada, 4. Rumbukwawa.

Three of the sites (Kofar Dundaye, Kofar Kware, Rumbukawa) are located in Sokoto North LGA and one site (Tudun Wada Dallatu) is in Sokoto South LGA. Both the LGAs were known high risk areas for poliovirus circulation.

\subsection{Collection of Sewage Samples}

Sewage samples were collected by trained local authorities. A sampling schedule of once per month per site was recommended in Kano. In Sokoto State, a sampling of twice per month per site was recommended. For each of the sampling site, 1 liter "grap" samples of the sewage were collected into clean, leak proof containers from the influent during the peak morning flow. Sewage samples were kept cool and were transported, usually within 1 day of collection, to the Ibadan national polio laboratory which is part of the GPLN.

\subsection{Extraction of Virus Particles from Sewage Samples}

The fluid grab samples were prepared for Poliovirus testing at the Ibadan Polio Laboratory. The Samples were concentrated using the two-phase separation method.

About $500 \mathrm{ml}$ of each specimen was centrifuged at $4{ }^{\circ} \mathrm{C}$ for 10 minutes at $1000 \mathrm{x} \mathrm{g}$. The $\mathrm{pH}$ of the supernatant was adjusted to $7.0-7.5$ before adding $287 \mathrm{ml}$ of $29 \%$ PEG 6000 , $39.5 \mathrm{ml}$ of $22 \%$ dextran and $35 \mathrm{ml} 5 \mathrm{M} \mathrm{NaCl}$ and then thoroughly mixed for $1 \mathrm{hr}$ using a magnetic stirrer. The mixture was then poured into a sterile $1 \mathrm{~L}$ separation funnel and left to stand overnight at $+4^{\circ} \mathrm{C}$ to give a phase separation. The entire lower phase was harvested and the interphase allowed to drop slowly into a sterile $50 \mathrm{ml}$ centrifuge tube. The harvested concentrate was finally used to resuspend the pellet kept after the initial centrifugation of the sample. Twenty percent chloroform was added to it, mixed thoroughly by vortexing and then centrifuged at $40^{\circ} \mathrm{C}$ for 20 minutes at $1500 \mathrm{~g}$. Antibiotics were added after chloroform extraction and aliquots then stored at $-20^{\circ} \mathrm{C}$ until inoculated. [WHO, 2003].

\subsection{Isolation of Polioviruses}

Isolation of Poliovirus was carried out in RD and L20B cell lines. Each $25 \mathrm{~cm} 3$ tissue culture flask of RD and L20B cells was inoculated with $0.5 \mathrm{ml}$ of specimen extract and incubated at $36^{\circ} \mathrm{C}$. Each inoculated flask was examined daily, using inverted microscope, for the appearance of cytopathic effect (CPE) for $5 \mathrm{~d}$. All positive flasks were kept at $-20^{\circ} \mathrm{C}$ while any culture positive in RD cells but negative in L20B cells was re-passaged in L20Bcells and examined for 5 days to ensure that they are actually not polioviruses [WHO, 2004]

\subsection{Neutralization Test}

Poliovirus identification was done using the micro neutralization techniques as described by WHO [2004]. Isolates were mixed with equal volumes of already prepared polyclonal anti-sera pools against poliovirus types 1, 2 and 3 supplied by RIVM. The mixtures were incubated for $1 \mathrm{~h}$ at $36^{\circ} \mathrm{C}$ to allow a possible reaction between the specific antibodies and the isolates. The micro-plates containing the cells suspension and viruses were observed daily for cytopathic effect (CPE). The antiserum pools that prevented the development of CPE indicated the identity of the virus isolate or mixture of viruses. Failure of a virus to replicate in the presence of a pool of anti-sera is due to the neutralization of infectivity by one of the anti-sera present in the pool

\subsection{Enzyme Linked Immuno-Sorbent Assay (ELISA) Method}

The RIVM ELISA for intra-typic differentiation was performed using single serotype isolates as described by WHO [2004]. RIVM supplied the kits containing essential reagents to carry out the ELISA method through WHO. Wells of micro-titer plates coated with bovine Immuno globulin $\mathrm{G}$ (IgG) antibodies to Polio virus types 1,2.and 3 were incubated with the identified and typed polioviruses strain to be tested. Incubation was the carried out with the typespecific, cross adsorbed rabbit antiserum. After washing off any unbound rabbit sera, peroxidase- labeled anti- rabbit $1 \mathrm{gG}$ antibody was added to detect bound rabbit sera. Substrate (TMB) was then added and incubated for 20 minutes at room temperature in the dark. The optical density (OD) of each well was measured at $450 \mathrm{~nm}$ using a spectrophotometer within $30 \mathrm{~min}$ after stopping of reaction.

\subsection{Probe hybridization Method for Intra-typic Differentiation}

RNA probe hybridization was performed using identified typed polioviruses of high titer as described by WHO [2004]. The Viral RNA was extracted and immobilized onto filters. Digoxygenin- labeled entero-virus group, Sabin type-specific and wild virus genotype-specific probes supplied by Centres for Disease Control and Prevention USA (CDC) in a kit through WHO were added and allowed to hybridize to the immobilize RNA. Unbound probe was removed by washing and bound probe was detected using a colorimetric reagent.

\subsection{Interpretation}

Group probe hybridized to all polioviruses and enteroviruses while Sabin specific probes hybridized to respective Sabin strains. The wild polioviruses formed hybrids with the corresponding wild genotype-specific probe. 


\subsection{Limitations}

Although the ability to isolate poliovirus from waste water in an endemic area has been demonstrated a number of times, much less is known about the sensitivity of these procedures for detecting low levels of poliovirus circulation. As a result of the poorly understood sensitivity, it will be difficult to interpret a negative finding from this project. If no polio virus is detected, is this because none is circulating or because the procedure is too insensitive to detect it? In light of this limitation, it is essential to interpret any negative result in the context of other epidemiological information, including the performance of AFP surveillance in the area. It is important to consider the findings from waste water samples as a supplement to the information gained through AFP surveillance rather than a replacement.

\section{Results}

In Kano State, from week 28 of 2011 to week 52 of 2012 , a total of 60 sewage samples were collected. Each of the three sites (Gogau Fagge sewage site (Fagge LGA), Jakara police station sewage site (Kano Municipal LGA) and Kurna Masalashi site (Fagge LGA) collected 20, 21 and 19 samples respectively.

In Sokoto State, from week 13 - 52 of 2012, a total of 80 sewage samples were collected. Each of the sites in Sokoto North (Kofar Dundaye site, Kofar Kware site and Rubun Kawa site) and, Tudun Wada Dallatu site in Sokoto South LGA collected 20 samples.

In Kano and Sokoto, 62 and 93 single or mixed isolates were detected from the samples. In Kano, 39 (63\%) of the isolates were Sabin viruses, 16 (26\%) were cVDPV2, 2 (3\%) were WPV1, $4(6 \%)$ were NPENT and $1(3 \%)$ were WPV3; table 1.

In Sokoto, 33 (35\%) of the isolates were cVDPV2, 27 (29\%) were Sabin viruses, 16 (17\%) were wild virus type 1 and $17(18 \%)$ were non polio enteroviruses, table 2 . No wild virus type 3 was detected from AFP cases and environmental samples in Sokoto State in 2012.

Table 1. Enteroviruses isolated in sewage samples of Kano State, Nigeria

\begin{tabular}{|c|c|c|c|c|c|c|}
\hline Site & cVDPV2 & Sabin & WPV1 & WPV3 & NPENT & Total \\
\hline Gogau Fagge site & 5 & 12 & 0 & 1 & 3 & 21 \\
\hline Jakara Police Station Site & 7 & 15 & 0 & 0 & 0 & 22 \\
\hline Kurna Masalashi site & 4 & 12 & 2 & 0 & 1 & 19 \\
\hline Isolates by type and total & 16 & 39 & 2 & 1 & 4 & 62 \\
\hline Percent isolates & $26 \%$ & $63 \%$ & $3 \%$ & $2 \%$ & $6 \%$ & $100 \%$ \\
\hline
\end{tabular}

Table 2. Enteroviruses isolated in sewage samples of Sokoto State, Nigeria

\begin{tabular}{|c|c|c|c|c|c|c|}
\hline Site & cVDPV2 & Sabin & WPV1 & WPV3 & NPENT & Total \\
\hline Kofar Dundaye site & 11 & 7 & 7 & 0 & 1 & 26 \\
\hline Kofar Kware site & 7 & 10 & 3 & 0 & 5 & 25 \\
\hline Runbukawa site & 7 & 5 & 5 & 0 & 6 & 23 \\
\hline Tudun Wada Dalatu site & 8 & 5 & 1 & 0 & 5 & 19 \\
\hline Isolates by type and total & 33 & 27 & 16 & 0 & 17 & 93 \\
\hline Percent isolates & $35 \%$ & $29 \%$ & $17 \%$ & $0 \%$ & $18 \%$ & $100 \%$ \\
\hline
\end{tabular}

Findings at each of the sewage sample collection sites:

\subsection{Kano Sites}

In Gogau Fagge sewage site, the laboratory detected 21 isolates of which, $12(57 \%)$ were Sabin viruses, 7 (33\%) cVDPV2 and $3(14 \%)$ were Non Polio Enterovirus and another $1(5 \%)$ was wild polio virus type three.

In Jakara police station sewage site, 22 viruses were isolated of which 13 (59\%) were Sabin viruses, 7(32\%) were cVDPV2. There was no Non polio entero-virus or wild polio that was isolated from this site.

In Kurna Masalashi site, 19 isolates were detected of which 12 (63\%) were Sabin viruses, 4 (21\%) were cVDPV2, two sample (11\%) had wild polio virus type 1 and one sample $(5 \%)$ had Non polio entero-virus.

\subsection{Sokoto Sites}

In Kofar Dundaye site there were 26 positive isolates of which 11 (42\%) were cVDPV2, 7(27\%) WPV1, 7 (27\%) Sabin viruses and 1 (4\%) was NPENT. Now WPV3 was detected.

In Kofar Kware site, there were 25 positive isolates; of which 10 (40\%) were Sabin viruses, 7 (28\%) were cVDPV2 and $3(12 \%)$ were wild polio virus type 1 and $5(20 \%)$ were NPENT. No WPV3 was detected.

In Rubun Kawa site, there were 23 positive isolates of which 7 (30\%) were cVDPV2, 5 (22\%) were wild polio virus type1, $5(22 \%)$ were Sabin and $6(26 \%)$ were NPENT. No WPV3 was detected.

In Tudun Wada Dallatu site, there were 19 positive isolates of which, $8(42 \%)$ were cVDPV2, $5(26 \%)$ were Sabin 
viruses, 1 (4\%) was wild polio virus type one, 5 (26\%) were NPENT. No WPV3 was detected.

The number of orphan polioviruses detected from environmental specimens in 2012 were two WPV1 from
Sokoto, (one each from Kofar Kware site and Rubun Kawa site), one WPV3 from Kano (Gagau Fagge site), and two cVDPV2 from Sokoto (one each from Tudun Wada Dallatu site and Kofar Kware site).

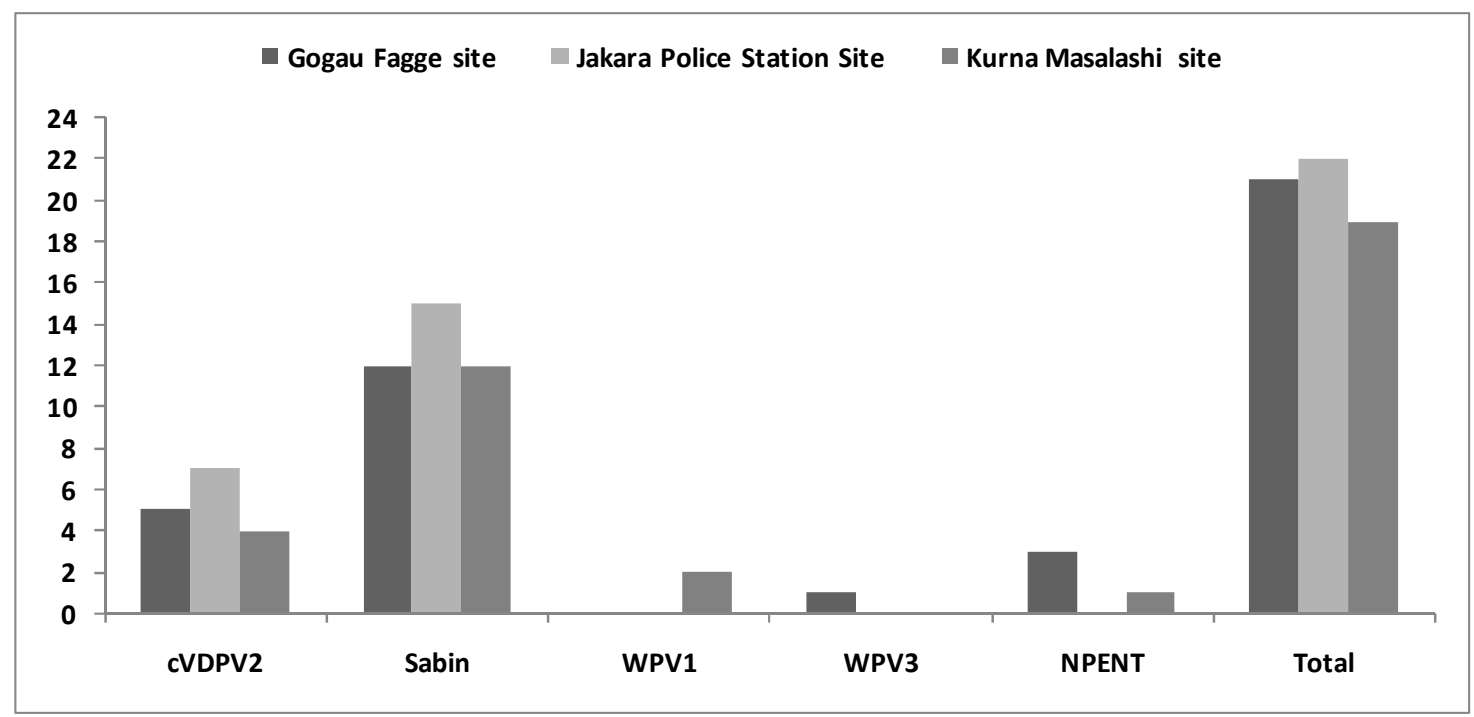

Figure 4. Isolates by type from environmental sites in Kano

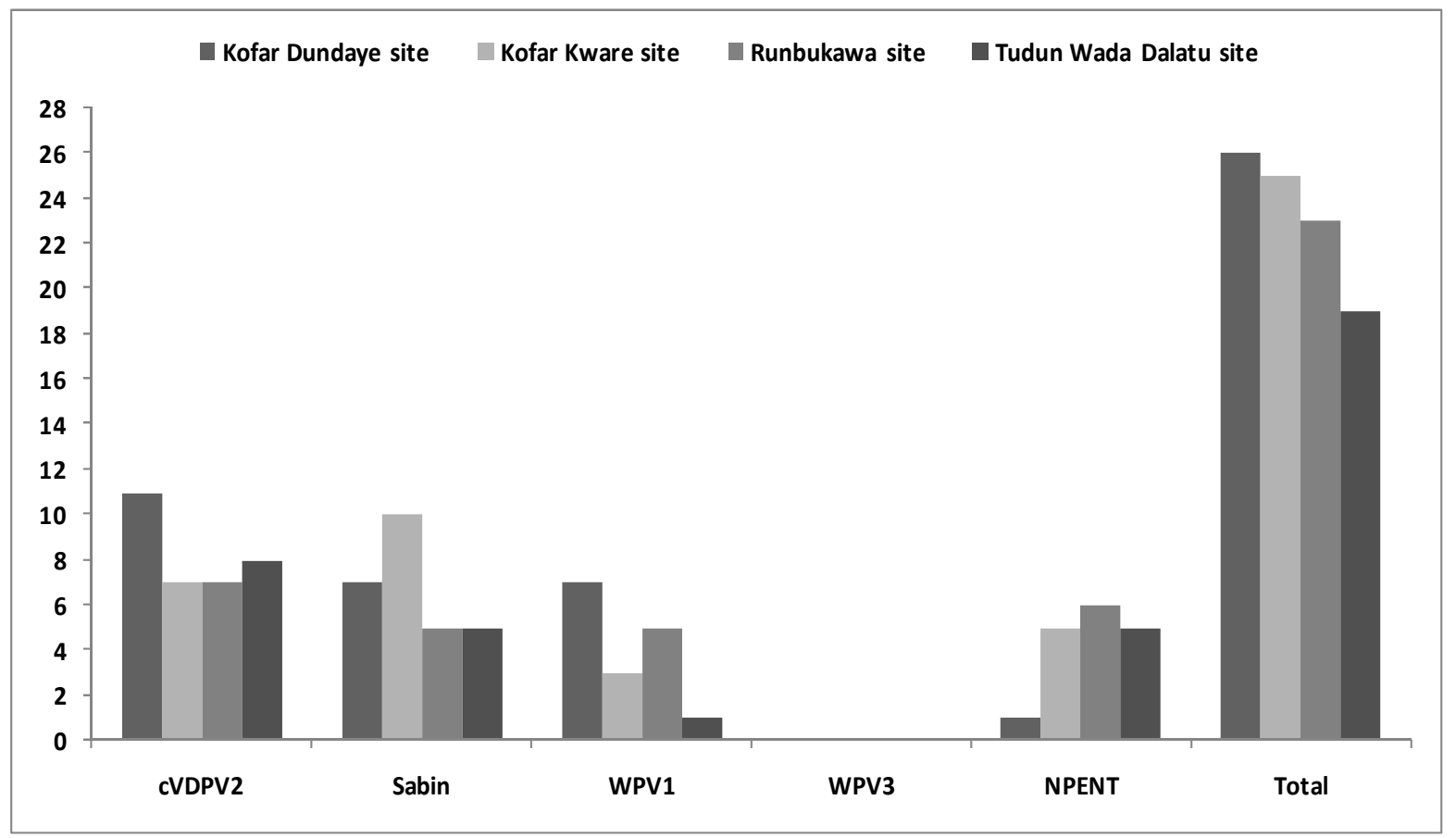

Figure 5. Isolates by type from environmental sites in Sokoto

\section{Discussion}

As at 8 February 2013, Nigeria reported a total of 122 cases of polio, from AFP cases, with onset in 2012 due to wild poliovirus, and a further $19 \mathrm{WPV}$ isolates from environmental samples. This represents a doubling of cases from the 62 (47 WPV1 \& 15 WPV3) reported in 2011. Most (104) of the cases detected in 2012 were due to wild poliovirus type 1 (WPV1), with 19 due to wild poliovirus type 3 [14]. There were also 8 cases of cVDPV2 from three States of Kano (3), Sokoto (3) and Kebbi (2).

In 2012, of the WPV isolates from environmental samples, 18 are WPV1, and 1 WPV3. Multiple isolates of cVDPV type 2 have also been reported, 8 from AFP cases and 47 from environmental samples ( 3 in Kano and 44 in Sokoto). The number of cVDPVs from AFP cases were significantly reduced in comparison to 2011[35]. However, significant numbers of cVDPVs were isolated from environmental samples especially in Sokoto State. 
Transmission of WPV in 2012 in Nigeria has been dominated by WPV1. While environmental sampling in Nigeria is not geographically representative, results are consistent with the serotype distribution in AFP cases, as WPV1 has been far more commonly detected than WPV3. In Sokoto State, no WPV3 was detected from both AFP and environmental samples.

The isolation rate of wild polio virus in the Kano sites (two WPV1 and one WPV3) is low. This finding supports the recommendation of the 22nd ERC on identification of new additional sites for polio environmental surveillance in Kano [18].

Most cVDPV2 isolates have been detected in Sokoto State; 3 from AFP cases/contacts and 44 from the environment. These isolates are all closely related, although their closest genetic relative was an environmental isolate from Kano in 2011.

There were areas with significant community-wide pockets of under-immunized children, who gave rise to sustained transmission in one LGA or clusters of LGAs over significant periods of time.

In addition to this sustained transmission, there was movement of virus over long distances, often across state and international borders, due to travelers and mobile/migrant populations; depending on where virus was carried, this can lead to sporadic cases, small outbreaks, or new sustained transmission pockets.

The Independent Monitoring Board (IMB) for global polio eradication initiative in its November 2012 report [19] indicated that population movement and poor immunity leaves a great number of other nations and areas at risk of importation, particularly Yemen, Libya, the Horn of Africa, Somalia, Ukraine, Uganda, and Kenya. Countries bordering Nigeria are deep of concern. They are tinder boxes that could all too easily be reignited.

The Nigeria National Polio Emergency Plan [20] included migrant strategy to reach nomadic children as one of the strategic priorities for polio eradication in Nigeria. The findings of the environmental surveillance re-emphasizes that the focus on high risk areas must be coupled with continued focus on migrant and mobile communities to reduce the risk of viral movement at the same time as immunity gaps are being closed in reservoir communities. The implementation of the Nigeria National Polio Emergency Plan is vital to achieve the goal of polio eradication

In Nigeria, AFP surveillance targets have continued to be met in all States. However, virological data indicated that surveillance was not sufficiently sensitive to detect all chains of WPV transmission in a timely manner [11, 16]. The detection of the orphan viruses in the environmental surveillance supported the need to improve surveillance sensitivity.

Supplementary environmental surveillance has been valuable in India, where low-level transmission has occurred as well as in Pakistan, where substantial AFP surveillance indicators have met targets but virologic analysis indicated major gaps in detection [8].
Similar to the findings in India and Pakistan [8] and in Iran [9] and in Israel [10], our study concludes that environmental surveillance is a very useful tool in complementing to the existing AFP surveillance system in Nigeria.

AFP surveillance data shows that cVDPV2 transmission has been very low in 2012 [14]. However, the findings of the environmental surveillance indicated an existence of populations groups with profound immunity gap towards type 2 polio who excreted the virus in the environment. In addition to the national polio campaigns using tOPV, an aggressive strategy should be adopted to mop up on any detection of cVDPV in cases, contacts, or the environment.

The environmental surveillance system should be scaled up in to other populated cities of Nigeria such as Lagos and Port Harcourt as well as security compromised areas such as Born State. New additional sites should also be considered in Kano State.

\section{Acknowledgement}

We thank the environmental health officers in Kano and Sokoto who collected the sewage samples; and the WHO staff in Kano and Sokoto States for coordinating the transportation of the samples to the national polio laboratory. Our appreciation is extended to the polio laboratory staff in Ibadan and the polio laboratory team in CDC, Atlanta, for sequencing the positive samples.

\section{Competing Interest}

The authors declare that they have no competing interest and did not receive any financial support.

\section{Authors' Contributions}

Goitom Weldegebriel conceived the study, participated in its design and performed the analysis and interpretation of the data and drafted the manuscript. . J Adeneji wrote the laboratory methods and directed the laboratory analysis. Gasasira, Okello, Elemuwa, Humayun, Diop and Mala contributed in the process of site identification, generation of raw data and in the write up of the manuscript.

\section{References}

[1] Pallansch MA, Roos RP [2001]. Enteroviruses Polioviruses, Coxsakieviruses, Echoviruses and Newer Enteroviruses, Field Virology. DM Knipe, PH Howley. Lippincott Williams \& Wilkins. $4^{\text {th }}$ ed. Philadelphia, PP. 685-775

[2] Semler BL, Wimmer E [2002]. Molecular Biology of Picornaviruses. $1^{\text {st }}$ American society for Microbiology. Washington.

[3] World Health Organization [2002]. Enteroviruses-non polio. Media center. Available at: http://www.who.int/mediacentre/factsheets/fs174/en. 
[4] Public Health Laboratory Network 2000. Polio laboratory case definition. Australian Government, Department of Health and Ageing. Available at: http://www.Health.gov.au/internet/wcms/publishing.nsf/Conte nt/cda-phlncd-polio.htm/\$File/polio.pdf.

[5] Polio laboratory network quarterly update, volume V1, number 2, W.H.O., May 2000; http://www.who.int/immunization_monitoring/62.pdf, accessed May 2012

[6] Fishing for polio, media room, Global Polio Eradication Initiative, $\quad 02 \quad$ April, 2012 http://www.polioeradication.org/Mediaroom/Newsstories/New sstories2012/tabid/461/iid/211/Default.aspx; accessed June 2012

[7] World Health Organization [2003]. Guidelines for environmental surveillance of polio virus circulation. Vaccines and Biologicals. (ordering code: WHO/V\&B/03.03] http://www.who.int/vaccinesdocuments/DocsPDF03/www737.pdf; accessed July 2011

[8] Centers for Disease Control and Prevention. Tracking progress Toward Global Polio Eradication ---World Wide, 2009-2010. MMWR Morbidity Mortality Weekly Report, 2011: 60 [14]; 441-446

[9] Khodael et al: environmental surveillance of polio and NonPolio enteroviruses in Sistan and Balouchestan Province. Iranian J publ Health, Vol.37, No3, 2008, pp.127-133

[10] Manor $\mathrm{Y}$ et al. Detection of poliovirus Circulation by environmental Surveillance in the Absence of Clinical cases in Israel and the Palestinian Authority. Journal of clinical microbiology, June 2009, P.1670-1675

[11] Weekly epidemiological record, World health Organization, progress towards eradicating poliomyelitis-Nigeria, January 2010 - June 2011, www.polioeradication.org/mdb.aspx;
[12] Centers for Disease Control and Prevention [2005]. Progress towards Poliomyelitis Eradication, Poliomyelitis Outbreak in Sudan, 2004. MMWR, 54 (4]97-9.

[13] Polio news, Global Polio Eradication Initiative, March edition, http://www.polioeradication.org/Portals/0/Document/Media/N ewsletter/PN201203_EN.pdf;

[14] National AFP database, FMoH, Nigeria.

[15] Global polio update, 30 May 2012 (email circulation to a global list).

[16] $19^{\text {th }}$ Meeting of the Expert Review Committee (ERC) meeting on polio eradication and routine immunization in Nigeria, Minna, Nigeria, 22-24 Sep, 2010

[17] Report of the $23^{\text {rd }}$ meeting of the Expert Review Committee (ERC) on polio eradication and routine immunization in Nigeria, Major findings and recommendations, Abuja, Nigeria, 28-29 March, 2012

[18] Report of the $24^{\text {th }}$ meeting of the Expert Review Committee (ERC) on polio eradication and routine immunization in Nigeria, Major findings and recommendations, Abuja, Nigeria, 10 - 11 September, 2012

[19] National Primary Health Care Development Agency, Nigeria National Polio eradication emergency plan, 2012.

[20] Polio's last stand? Report of the Independent monitoring board of the global polio eradication initiative, November 2012.

http://www.polioeradication.org/Portals/0/Document/Aboutus/ Governance/IMB/7IMBMeeting/7IMB_Report_EN.pdf. 